\title{
Impact of sites versus number of metastases on survival of patients with organ metastasis from newly diagnosed cervical cancer
}

This article was published in the following Dove Press journal: Cancer Management and Research

\author{
Zhuomin Yin ${ }^{1,2}$ \\ Huarong Tang' \\ $\mathrm{Li} \mathrm{Li}{ }^{1}$ \\ Juan $\mathrm{Ni}^{1}$ \\ Shuhui Yuan' \\ Hanmei Lou' \\ Ming Chen ${ }^{2}$
}

'Department of Gynecologic Radiation Oncology, Zhejiang Cancer Hospital, Hangzhou 310022, People's Republic of China; ${ }^{2}$ Department of Radiation Oncology (Zhejiang Key Laboratory of Radiation Oncology), Zhejiang Cancer Hospital, Hangzhou 310022, People's

Republic of China
Correspondence: Ming Chen

Department of Radiation Oncology,

Zhejiang Cancer Hospital, No. I Banshan

East Road, Hangzhou 310022, People's

Republic of China

$\mathrm{Tel}+8657188128172$

Fax +8657188122508

Email chenmingdr@I63.com
Objective: The aim of this study was to investigate the potential associations of the sites and the number of specific metastases with survival in patients newly diagnosed with cervical cancer.

Methods: Medical records of patients with organ metastases of newly diagnosed cervical cancer at Zhejiang Cancer Hospital from October 2006 to December 2016 were reviewed retrospectively. Survival times were compared using the Kaplan-Meier method. Variables associated with survival were identified using univariate and multivariate Cox proportional hazards models.

Results: A total of 99 patients with newly diagnosed organ metastatic cervical cancer were identified. Median follow-up was 11.6 months (range, 0.5-114.7 months). Median overall survival (OS) time was 11.7 months from diagnosis, with 1, 2, and 5-year OS rates of $48.2 \%$, $22.8 \%$, and $12.6 \%$, respectively. The most common site of organ metastasis was bone $(36.8 \%)$, followed by lung $(32.8 \%)$ and liver $(24 \%)$. In univariate analysis, OS rates were better for bone metastasis than visceral metastasis $(P=0.013)$, oligometastasis than nonoligometastasis $(P=0.003)$ and single organ metastasis than multiple organ metastases $(P=0.016)$, while that for liver metastasis was poorer than non-liver metastases $(P<0.001)$. In multivariate analysis, liver metastasis (hazard ratio [HR] $=4.02 ; 95 \%$ confidence interval $[\mathrm{CI}], 1.15-14.05, P=0.029)$ was significantly and independently related to poor overall survival.

Conclusion: Our data revealed the site of metastasis is associated with overall survival of patients with newly diagnosed organ metastatic cervical cancer, with liver metastasis signifying particularly poor overall survival. Individualized treatments should be administered to patients depending on the specific metastatic sites.

Keywords: uterine cervical neoplasm, survival, organ metastasis, metastatic site, number of metastases

\section{Introduction}

Cervical cancer is the fourth most common female malignancy in terms of both incidence and mortality, with an estimated 569,847 new cases and 311,365 deaths worldwide recorded in $2018 .^{1}$ Two types of metastasis, specifically, lymph node and organ, have been identified to date. Metastatic cervical cancer has a poor prognosis with a median survival time of only 8 to 13 months. ${ }^{2}$ Prognosis of patients with organ metastasis is weaker than that of patients with lymph node metastasis. Notably, organ metastasis is associated with a 5.3-fold higher risk of death, 
compared to lymphatic metastasis. ${ }^{3-5}$ To date, limited advances have been made in the understanding of cervical cancer with organ metastasis due to small sample sizes. ${ }^{6}$

Around $3 \%$ of patients have stage IVB uterine cervical cancer, with lymph nodal metastasis accounting for the majority and organ metastasis for a small proportion of cases. $^{7}$ The most common metastatic sites of cervical cancer are lung, bone, liver, and brain, with possible involvement of other places. ${ }^{8}$ The majority of studies to date have focused on a single metastasis site or mixed nodal and organ metastasis. ${ }^{4,5}$ However, reports on newly diagnosed organ metastatic cervical cancer are rare, and these cases require further investigation.

The primary objective of this study was to determine the characteristics of different metastatic organs and assess the relationships between specific sites of metastasis (bone, lung, and liver) and the number of metastatic lesions (oligometastasis and single organ metastasis) and survival in cases of newly diagnosed cervical cancer with organ metastasis.

\section{Materials and methods}

\section{Patients}

Patients diagnosed with primary organ metastatic cervical cancer treated at Zhejiang Cancer Hospital from October 2006 to December 2016 were included. Primary uterine cervical cancer was confirmed in all patients via pathological biopsy. Metastatic lesions were confirmed firstly through imaging techniques $(47,47.5 \%)$, such as magnetic resonance imaging (MRI), computed tomography (CT) and emission computed tomography (ECT), secondly via positron emission tomography-computed tomography (PET-CT) $(31,31.3 \%)$, and finally via histopathological or cytopathological examination $(21,12.2 \%)$ for metastatic lesions that could not be confirmed by imaging. All examinations were based on the opinions of the physician team. For some complicated cases, decisions are made through multidisciplinary discussions. Lymph node metastasis was defined based on CT showing $>10 \mathrm{~mm}$ enlargement in the short axis. All patients were clinically diagnosed as primary cervical cancer with organ metastasis. We excluded four patients for whom cervical cancer was not the first malignancy or information on variables was unavailable.

The study was approved by the Institutional Review Board and Ethics Committee of Zhejiang Cancer Hospital (IRB-2017-94). This study was conducted in accordance with the Declaration of Helsinki. Written informed consent for the collection of medical information was obtained from all participants. All procedures performed in the study were in accordance with the ethical standards of the institutional research committee.

\section{Variables}

The medical records of all patients were reviewed retrospectively, and variables analyzed at the time of initial diagnosis (age, size of cervical tumor, histological subtype, Eastern Cooperative Oncology Group performance status (ECOG PS), site of metastasis (bone, lung and liver), number of metastatic sites (oligometastasis, non-oligometastasis, single organ or multiple organ metastasis), distant lymph node metastases, initial treatment modalities and survival data were collected. The primary endpoint of the current study was overall survival (OS). Progression-free survival (PFS) and OS times were defined from the date of diagnosis.

In total, data on 11 variables were obtained for survival analysis. Ten variables had binary classification while therapeutic variables were divided into four categories (chemotherapy combined with local treatment (radiotherapy or operation for the local pelvic tumor or metastatic lesions), chemotherapy, radiotherapy, and supportive care). Chemotherapeutic regimens mainly involved cisplatin $\left(60-70 \mathrm{mg} / \mathrm{m}^{2}\right)$ plus paclitaxel $\quad\left(135-150 \quad \mathrm{mg} / \mathrm{m}^{2}\right) \quad(\mathrm{TP})$ and carboplatin $($ AUC $=5)$ plus paclitaxel $\left(135-150 \mathrm{mg} / \mathrm{m}^{2}\right)$ (TC). Organ metastasis was defined as the metastasis from cervical cancer involving one or more distant organs, such as bone, lung or liver. Oligometastasis was defined as limited a single organ with $\leq$ three metastatic lesions. Single organ metastasis was defined in cases where all metastases were confined to an only organ and multiorgan metastasis defined as $\geq 2$ organs. Lymph node metastasis beyond the pelvic space was classified as distant lymph node metastasis.

\section{Statistical analysis}

Survival times between groups were compared based on the log-rank test of Kaplan-Meier analysis. Prognostic factors for PFS and OS were identified using univariate and multivariate Cox proportional hazards regression analyses. All survival data were analyzed with SPSS version 22 statistical software (IBM Corp., version 22.0; Armonk, NY, USA). $P$-values $<0.05$ were considered statistically significant. 


\section{Results}

\section{Patients and tumor characteristics}

In total, 99 women with organ metastatic cervical cancer were reviewed. Clinicopathological characteristics of patients are summarized in Table 1 . The median age was

Table I Patient baseline characteristics at the time of initial diagnosis and treatment types $(n=99)$

\begin{tabular}{|c|c|}
\hline Characteristics & No.\% \\
\hline Age (yr), median (range) & $53(29-90)$ \\
\hline \multicolumn{2}{|l|}{ ECOG PS } \\
\hline 0 & $35(35.4)$ \\
\hline I & $16(16.2)$ \\
\hline 2 & $22(22.2)$ \\
\hline 3 & $20(20.2)$ \\
\hline 4 & $6(6.1)$ \\
\hline \multicolumn{2}{|l|}{ Histological subtype } \\
\hline Squamous cell carcinoma & $80(80.8)$ \\
\hline Small-cell neuroendocrine carcinoma & $7(7.1)$ \\
\hline Adenocarcinoma & $6(6.1)$ \\
\hline Adeno-squamous cell carcinoma & $3(3)$ \\
\hline Undifferentiated carcinoma & $2(2)$ \\
\hline Sarcoma & $I(I)$ \\
\hline \multicolumn{2}{|l|}{ Primary tumor size $(\mathrm{cm})$} \\
\hline$\geq 4-\mathrm{cm}$ & 72 \\
\hline$<4-\mathrm{cm}$ & 27 \\
\hline \multicolumn{2}{|l|}{ Initial treatment } \\
\hline Chemotherapy + local treatment & $49(49.5)$ \\
\hline Chemotherapy & $15(15.2)$ \\
\hline Radiotherapy & II (II.I) \\
\hline Supportive care & $24(24.2)$ \\
\hline \multicolumn{2}{|l|}{ Diagnostic methods of metastatic lesions } \\
\hline $\mathrm{CT} / \mathrm{MRI} / \mathrm{ECT}$ & $47(47.5)$ \\
\hline PET-CT & $31(31.3)$ \\
\hline Biopsy & $2 \mid(2 \mid .2)$ \\
\hline \multicolumn{2}{|l|}{ Single organ metastasis } \\
\hline Bone & $24(24.2)$ \\
\hline Lung & $16(16.2)$ \\
\hline Liver & $3(3)$ \\
\hline Brain & I (I) \\
\hline Spleen & $I(1)$ \\
\hline \multicolumn{2}{|l|}{ Single organ + distant lymph node metastasis } \\
\hline Bone + distant lymph node & $12(2||)$. \\
\hline Lung + distant lymph node & $10(10.1)$ \\
\hline Liver + distant lymph node & $10(10.1)$ \\
\hline \multicolumn{2}{|l|}{ Two organ metastasis } \\
\hline Liver + lung & $3(3)$ \\
\hline
\end{tabular}

(Continued)
Table I (Continued).

\begin{tabular}{|l|l|}
\hline Characteristics & No.\% \\
\hline $\begin{array}{l}\text { Lung + spleen } \\
\text { Liver + bone } \\
\text { Liver + kidney }\end{array}$ & $2(2)$ \\
I (I) \\
\hline $\begin{array}{l}\text { Two organ metastasis + distant lymph node } \\
\text { metastasis }\end{array}$ \\
$\begin{array}{l}\text { Liver + lung + distant lymph node } \\
\text { Bone + lung + distant lymph node } \\
\text { Liver + bone + distant lymph node } \\
\text { Liver + adrenal gland + distant lymph node }\end{array}$ \\
\hline $\begin{array}{l}\text { Multiorgan metastasis } \\
\text { Liver + lung + bone + breast }\end{array}$ & $\begin{array}{l}6(6.1) \\
\text { I (I) }\end{array}$ \\
\hline $\begin{array}{l}\text { Multiorgan + distant lymph node metastasis } \\
\text { Liver + lung + bone + adrenal gland + distant } \\
\text { lymph node }\end{array}$ & $\mathrm{I}(\mathrm{I})$ \\
\hline
\end{tabular}

Abbreviations: ECOG PS, Eastern Cooperative Oncology Group performance status; MRI, magnetic resonance imaging; CT, computed tomography; ECT, emission computed tomography; PET-CT, positron emission tomography-computed tomography.

53 years (range, 29-90 years). A total of 75 patients (75.8\%) received treatment, among these patients, 64 $(85.3 \%)$ received systemic chemotherapy, and $60(80 \%)$ received radiotherapy for pelvic local and/or metastatic lesions. Twenty-four patients $(24.2 \%)$ received supportive care, $16(16.2 \%)$ had poor performance status or reduced organ function, with no tolerance to chemotherapy and radiotherapy, and $8(8.1 \%)$ refused both chemotherapy and radiotherapy.

\section{Characteristics of organ metastasis}

Distribution of metastatic sites is shown in Table 1. Within the 99 patients, a total of 125 metastatic organ sites were identified, the most common being bone (46, 36.8\%), followed by lung $(41,32.8 \%)$ and liver $(30,24 \%)$. The numbers of organ metastatic lesions were classified as follows: oligometastasis $(37,37.3 \%)$, non-oligometastasis $(62,62.6 \%)$, single organ metastasis $(77,77.8 \%)$ and multiorgan metastasis $(22,22.2 \%)$. In patients with bone, lung and liver metastasis, the incidence of oligometastasis was $58.7 \%, 9.8 \%$, and $13.3 \%$, and the incidence of single organ metastasis was $80.4 \%, 63.4 \%$, and $50 \%$, respectively.

\section{Survival analysis}

Overall survival curves for all patients are presented (Figure 1A). The median follow-up period for the whole 

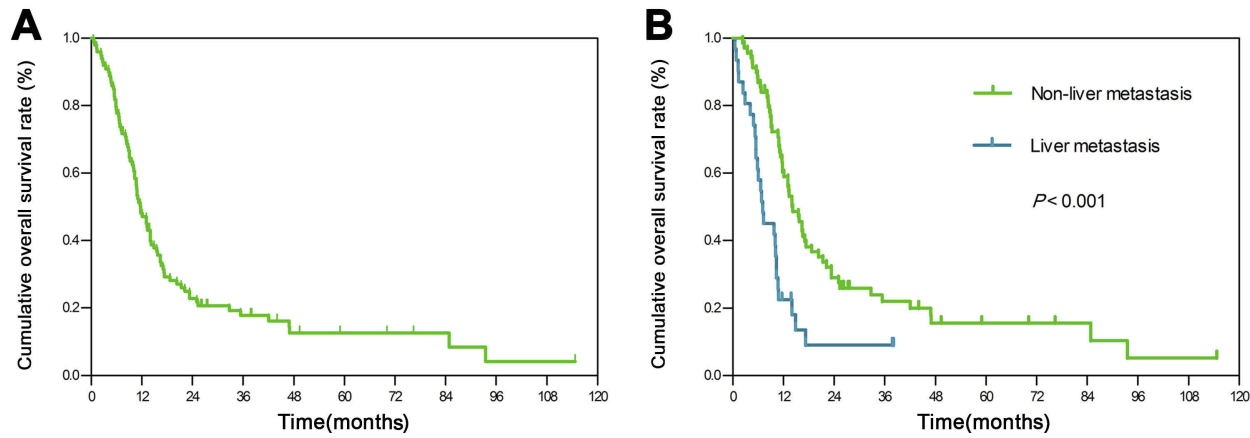

Figure I Overall survival among patients with organ metastatic cervical cancer at diagnosis.

Notes: Kaplan-Meier curves of overall survival for (A) patients in the whole group, (B) liver metastasis vs non-liver metastasis.

group was 11.6 months (range, 0.5-114.7 months) after clinical diagnosis. Median OS was 11.7 months, with 1-, 2 -, and 5-year rates of $48.2 \%, 22.8 \%$, and $12.6 \%$, respectively. Median PFS was 5.6 months, with 1-, 2-, and 5-year rates of $26.3 \%, 16.7 \%$, and $10.6 \%$, respectively. Table 2 shows median survival time, 1- and 2-year overall survival rates for all variables. Median OS, median PFS, 1- and 2year OS rates showed a gradually decreasing trend for patients with oligometastasis, bone, single organ, lung and liver metastasis.

The results of univariate analyses are summarized in Table 3. Overall survival rates of patients with non-liver metastasis were better than those with liver metastasis $(P<0.001)$ (Figure 1B), as were those with bone metastasis compared to those with visceral metastasis $(P=0.013)$, those with oligometastasis compared to those with nonoligometastasis $(P=0.003)$ and those with single organ metastasis compared to those with multiple organ metastases $(P=0.016)$.

The results of multivariate analyses are shown in Table 4. Liver metastasis was an independent prognostic factor for OS (hazard ratio $[\mathrm{HR}]=4.02,95 \%$ confidence interval $[\mathrm{CI}], 1.15-14.05, P=0.029)$ and PFS (HR $=3.77$, 95\% CI, 1.22-11.69, $P=0.021)$. Moreover, chemotherapy combined with local treatment $(\mathrm{HR}=0.40,95 \% \mathrm{CI}, 0.19-$ $0.82, P=0.012$ ) was associated with better OS and chemotherapy combined with local treatment (HR $=0.42,95 \%$ CI, $0.20-0.91, P=0.027$ ) positively affected the PFS.

\section{Discussion}

Data from the current study revealed distinct prognosis of cervical cancer patients with different organ metastatic sites. Liver metastasis was identified as an independent prognostic factor affecting overall survival. Furthermore, longer OS and PFS were associated with bone metastasis compared with visceral metastases, oligometastasis compared with non-oligometastasis, and single organ metastasis compared with multiple organ metastases.

Cervical cancer with organ metastasis at the time of diagnosis is relatively rare. In our experiments, bone $(36.8 \%)$ was the most common metastatic site, followed by lung $(32.8 \%)$ and liver $(24 \%)$. An earlier retrospective study on 316 patients with organ metastatic cervical cancer, including metastases at initial diagnoses and after treatment, showed that the most common sites are lung (126, 39.9\%), bone $(85,26.9 \%)$, and liver $(48,15.2 \%){ }^{8}$ Another investigation demonstrated that lung is the most common site of metastasis, followed by bone and liver. ${ }^{9,10}$ However, we observed a higher incidence of bone metastasis than lung metastasis, inconsistent with previous reports. This discrepancy may be attributable to two reasons: firstly, previous reports included organ metastasis at initial diagnosis and after treatment, whereas our study included organ metastases at initial diagnosis only; and secondly, advanced image diagnosis technologies, such as PET-CT, used in this study are more sensitive in the detection of early bone metastases than traditional imaging techniques, such as CT and MRI used in previous studies. 8,11

Limited studies to date have focused on the potential association between the sites or the number of organ metastases and prognosis in newly diagnosed cervical cancer although this issue is currently under exploration in other solid tumors. A retrospective study with a large sample size performed to assess the relationship between sites and number of metastases and overall survival in primary ovarian cancer showed that the locations of distant metastases rather than number of metastases affect overall survival of patients. ${ }^{12}$ Another retrospective study on stage IV non-small cell lung cancer proposed that the number rather than sites of extrapulmonary metastases is closely associated with prognosis of 
Table 2 Median survival time and survival rates according to clinicopathologic factors $(\mathrm{n}=99)$

\begin{tabular}{|c|c|c|c|c|c|}
\hline \multirow[t]{2}{*}{ Variable } & \multirow[t]{2}{*}{ No. } & Median OS & Median PFS & I-year OS rates & 2-year OS rates \\
\hline & & (Months) & (Months) & (\%) & (\%) \\
\hline \multicolumn{6}{|l|}{ Age } \\
\hline$\geq 50$ years & 65 & 11.7 & 6.4 & 47.1 & 24.5 \\
\hline$<50$ years & 34 & 10.7 & 4.6 & 50 & 19.3 \\
\hline \multicolumn{6}{|l|}{ Size of the cervical tumor } \\
\hline$\geq 4-\mathrm{cm}$ & 72 & 11.7 & 5.2 & 48.6 & 21.7 \\
\hline$<4-\mathrm{cm}$ & 27 & 11.6 & 8.1 & 46.9 & 25.8 \\
\hline \multicolumn{6}{|l|}{ Histological subtype } \\
\hline SCC & 80 & 13.0 & 5.7 & 52.5 & 25.9 \\
\hline Non-SCC & 19 & 8.3 & 3.9 & 29.2 & 8.8 \\
\hline \multicolumn{6}{|l|}{ ECOG PS } \\
\hline $0-2$ & 73 & 14.8 & 7.5 & 59.9 & 29.6 \\
\hline $3-4$ & 26 & 5.8 & 1.9 & 15.4 & 3.8 \\
\hline \multicolumn{6}{|l|}{ Oligometastasis } \\
\hline Yes & 36 & 16.6 & 9.3 & 66.7 & 41.2 \\
\hline No & 63 & 10.1 & 4.3 & 37.5 & 12 \\
\hline \multicolumn{6}{|l|}{ Bone metastasis } \\
\hline Yes & 46 & 14.8 & 7.1 & 65.2 & 31.1 \\
\hline No & 53 & 9.7 & 4.3 & 33.1 & 15.6 \\
\hline \multicolumn{6}{|l|}{ Number of organ metastasis } \\
\hline Single & 77 & 13.4 & 6.9 & 55.5 & 27.4 \\
\hline Multiple & 22 & 10.0 & 4.3 & 22.7 & 5.7 \\
\hline \multicolumn{6}{|l|}{ Lung metastasis } \\
\hline Yes & 41 & 10.8 & 4.6 & 35.4 & 12.6 \\
\hline No & 58 & 14.0 & 6.5 & 56.9 & 30 \\
\hline \multicolumn{6}{|l|}{ Liver metastasis } \\
\hline Yes & 30 & 6.8 & 3.7 & 20 & 8 \\
\hline No & 69 & 14.2 & 7.5 & 60.5 & 29.1 \\
\hline \multicolumn{6}{|l|}{ Distant lymph node metastasis } \\
\hline Yes & 46 & 10.9 & 4.7 & 43.5 & 15.6 \\
\hline No & 53 & 13.1 & 6.0 & 52.2 & 29 \\
\hline \multicolumn{6}{|l|}{ Initial treatment } \\
\hline Chemotherapy+local treatment & 49 & 17.3 & 9.4 & 64.8 & 37 \\
\hline Chemotherapy & 15 & 10.7 & 5.9 & 40 & 8.9 \\
\hline Radiotherapy & 11 & 5.9 & 2.4 & 27.3 & 9.1 \\
\hline Supportive care & 24 & 7.3 & 1.8 & 29.2 & 8.3 \\
\hline
\end{tabular}

Abbreviations: SCC, Squamous cell carcinoma; ECOG PS, Eastern Cooperative Oncology Group performance status; OS, overall survival; PFS, progression-free survival.

patients. ${ }^{13}$ Accordingly, we investigated the relationship between locations or the number of metastases and survival in cervical cancer in the current study. Here, we mainly focused on the effects of three main metastatic organs (bone, lung, and liver) and two paired metastatic site number-related variables (oligometastasis vs non-oligometastasis and single organ metastasis vs multiple organ metastases) on overall patient survival.

Numerous studies have confirmed that the number of metastatic lesions and metastatic organ sites affects prognosis. ${ }^{13-15}$ However, the impact of these factors on the prognosis of patients with primary cervical cancer has not been documented as yet. In the current investigation, patients with oligometastasis showed a survival advantage over the non-oligometastasis group in terms of OS and PFS. Furthermore, patients with single organ metastasis had a survival benefit over those with multiple organ metastases in terms of OS. Although these were not independent prognostic factors for OS, our results suggest that cervical cancer involving fewer metastatic lesions or organs is associated with better prognosis and these patients are thus more likely to benefit from aggressive treatment. 
Table 3 Univariate analysis of prognostic factors for progression-free survival and overall survival $(n=99)$

\begin{tabular}{|c|c|c|c|c|}
\hline \multirow[t]{2}{*}{ Variable } & \multicolumn{2}{|l|}{ PFS } & \multicolumn{2}{|l|}{ os } \\
\hline & HR (95\% Cl) & $P$-value & HR (95\% Cl) & $P$-value \\
\hline \multicolumn{5}{|l|}{ Age } \\
\hline$\geq 50$ years & 1 & & I & \\
\hline$<50$ years & $1.34(0.86-2.09)$ & 0.198 & $1.26(0.8 \mathrm{I}-1.96)$ & 0.310 \\
\hline \multicolumn{5}{|l|}{ Size of the cervical tumor } \\
\hline$\geq 4-\mathrm{cm}$ & 1 & & 1 & \\
\hline$<4-\mathrm{cm}$ & $0.86(0.52-1.42)$ & 0.541 & $0.86(0.52-1.43)$ & 0.554 \\
\hline \multicolumn{5}{|l|}{ Histological subtype } \\
\hline $\mathrm{SCC}$ & 1 & & I & \\
\hline Non-SCC & $1.39(0.8 \mathrm{I}-2.38)$ & 0.234 & $1.86(1.08-3.21)$ & 0.026 \\
\hline \multicolumn{5}{|l|}{ ECOG PS } \\
\hline $0-2$ & 1 & & 1 & \\
\hline $3-4$ & $4.14(2.52-6.79)$ & $<0.001$ & $3.29(2.04-5.32)$ & $<0.001$ \\
\hline \multicolumn{5}{|l|}{ Oligometastasis } \\
\hline Yes & 1 & & I & \\
\hline No & $2.10(1.32-3.35)$ & 0.002 & $2.04(1.28-3.24)$ & 0.003 \\
\hline \multicolumn{5}{|l|}{ Bone metastasis } \\
\hline Yes & 1 & & I & \\
\hline No & $1.72(1.10-2.67)$ & 0.017 & $1.74(1.13-2.70)$ & 0.013 \\
\hline \multicolumn{5}{|l|}{ Number of organ metastasis } \\
\hline Single & I & & I & \\
\hline Multiple & $1.58(0.95-2.62)$ & 0.08 & $1.89(1.13-3.17)$ & 0.016 \\
\hline \multicolumn{5}{|l|}{ Lung metastasis } \\
\hline Yes & 1 & & I & \\
\hline No & $0.62(0.40-0.96)$ & 0.033 & $0.66(0.43-1.02)$ & 0.060 \\
\hline \multicolumn{5}{|l|}{ Liver metastasis } \\
\hline Yes & I & & I & \\
\hline No & $0.48(0.30-0.76)$ & 0.002 & $0.39(0.24-0.62)$ & $<0.001$ \\
\hline \multicolumn{5}{|l|}{ Distant lymph node metastasis } \\
\hline Yes & 1 & & I & \\
\hline No & $0.66(0.43-1.02)$ & 0.061 & $0.65(0.42-1.01)$ & 0.050 \\
\hline \multicolumn{5}{|l|}{ Initial treatment } \\
\hline Supportive care & 1 & & 1 & \\
\hline Chemotherapy + local treatment & $0.27(0.16-0.45)$ & $<0.001$ & $0.31(0.18-0.53)$ & $<0.001$ \\
\hline Chemotherapy & $0.52(0.27-1.02)$ & 0.056 & $0.74(0.38-1.44)$ & 0.378 \\
\hline Radiotherapy & $1.29(0.62-2.68)$ & 0.499 & $0.98(0.48-2.03)$ & 0.973 \\
\hline
\end{tabular}

Abbreviations: SCC, Squamous cell carcinoma; ECOG PS, Eastern Cooperative Oncology Group performance status; Cl, confidence interval; HR, hazard ratio; OS, overall survival; PFS, progression-free survival.

Since liver metastases are rare (1.2-2.2\%), limited reports to date have focused on liver metastases from cervical cancer. ${ }^{16}$ In an earlier investigation, primary liver metastasis accounted for $10 \%$ and recurrent liver metastasis for $90 \%$ cases, only one case $(5 \%)$ was an isolated liver metastasis, the prognosis was inferior, median survival time was ten months, and no patients lived beyond two years. ${ }^{17}$ In our study, the incidence of multiple organ metastasis for patients with bone, lung, liver metastases was $19.6 \%, 36.6 \%$, and $50 \%$, respectively. Median OS and median PFS for patients with liver metastasis were 6.8 and 3.7 months, respectively, which were 
Table 4 Multivariate analysis of prognostic factors for progression-free survival and overall survival $(n=99)$

\begin{tabular}{|c|c|c|c|c|}
\hline \multirow[t]{2}{*}{ Variable } & \multicolumn{2}{|c|}{ PFS } & \multicolumn{2}{|c|}{ os } \\
\hline & HR (95\% Cl) & $P$-value & HR (95\% CI) & $P$-value \\
\hline \multicolumn{5}{|l|}{ Histological subtype } \\
\hline SCC & 1 & & 1 & \\
\hline Non-SCC & $1.30(0.70-2.4 \mathrm{I})$ & 0.404 & $1.78(0.94-3.37)$ & 0.077 \\
\hline \multicolumn{5}{|l|}{ ECOG PS } \\
\hline $0-2$ & 1 & & I & \\
\hline $3-4$ & $1.91(0.88-4.14)$ & 0.102 & $1.48(0.70-3.13)$ & 0.300 \\
\hline \multicolumn{5}{|l|}{ Oligometastasis } \\
\hline Yes & 1 & & I & \\
\hline No & $1.39(0.75-2.56)$ & 0.292 & $1.27(0.69-2.37)$ & 0.446 \\
\hline \multicolumn{5}{|l|}{ Bone metastasis } \\
\hline Yes & 1 & & I & \\
\hline No & $2.32(0.85-6.38)$ & 0.101 & $1.88(0.62-5.76)$ & 0.268 \\
\hline \multicolumn{5}{|l|}{ Number of organ metastasis } \\
\hline Single & 1 & & 1 & \\
\hline Multiple & $2.70(0.86-8.43)$ & 0.088 & $2.01(0.57-7.03)$ & 0.275 \\
\hline \multicolumn{5}{|l|}{ Lung metastasis } \\
\hline Yes & 1 & & I & \\
\hline No & $0.4 I(0.15-1.12)$ & 0.082 & $0.6 \mathrm{I}(0.20-1.83)$ & 0.375 \\
\hline \multicolumn{5}{|l|}{ Liver metastasis } \\
\hline Yes & $3.77(1.22-11.69)$ & 0.021 & $4.02(1.15-14.05)$ & 0.029 \\
\hline No & 1 & & 1 & \\
\hline \multicolumn{5}{|l|}{ Distant lymph node metastasis } \\
\hline Yes & 1 & & 1 & \\
\hline No & $0.97(0.60-1.56)$ & 0.892 & $0.87(0.53-1.43)$ & 0.575 \\
\hline \multicolumn{5}{|l|}{ Initial treatment } \\
\hline Supportive care & 1 & & 1 & \\
\hline Chemotherapy+ local treatment & $0.42(0.20-0.91)$ & 0.027 & $0.40(0.19-0.82)$ & 0.012 \\
\hline Chemotherapy & $0.67(0.28-I .58)$ & 0.353 & $0.74(0.33-1.67)$ & 0.467 \\
\hline Radiotherapy & $1.45(0.68-3.10)$ & 0.341 & $1.21(0.54-2.72)$ & 0.637 \\
\hline
\end{tabular}

Abbreviations: SCC, Squamous cell carcinoma; ECOG PS, Eastern Cooperative Oncology Group performance status; Cl, confidence interval; HR, hazard ratio; OS, overall survival; PFS, progression-free survival.

shorter than those for patients with lung or bone metastasis. In a comparative analysis, patients with liver metastasis showed the highest incidence of multiple organ metastasis and shortest survival time among the three groups (bone, lung, and liver). The characteristics of disseminated metastasis may be the cause of poor prognosis, supporting liver metastasis as an independent prognostic factor for OS and PFS.

A recent study compared the survival of 12 cervical cancer patients with bone metastasis only to 43 cases of visceral metastasis (liver and lung). The results showed that prognosis of bone metastasis was better than that of visceral metastasis with median OS times of 14 and 9 months $(p=0.014)$, respectively. ${ }^{18}$ Consistent with previous findings, in our study, the prognosis of patients with bone metastasis was better than that of patients with visceral metastasis, with median OS times of 14.8 and 9.7 months, respectively $(p=0.012)$. We infer that better prognosis of patients with bone metastasis is attributable to the higher proportion of oligometastasis (58.7\%) and single organ metastasis (80.4\%) in this patient group relative to those with visceral metastases.

It is essential to acknowledge that our study has some limitations. First, the study design was a retrospective review of a small sample size at a single cancer center, and thus potential bias could not be avoided. Second, the heterogeneity of patient characteristics and treatments might be a confounding factor. Finally, owing to the long period of the study, potential bias due to differences in diagnostic and therapeutic techniques could not be excluded. Further prospective multicenter studies are necessary to reduce bias.

Despite the above drawbacks, our results support the association of specific metastasis organ sites with prognosis. 
Liver metastasis was identified as an independent prognostic factor related to poor overall survival. Moreover, the involvement of a lower number of metastatic lesions or organs was indicative of better prognosis. Cervical cancer patients with organ metastasis characteristics associated with good prognosis might benefit more from individualized treatment modalities, leading to higher survival benefits.

\section{Acknowledgment}

The study was supported by a grant from the Zhejiang Medical Science and Technology Foundation (No. 2018RC022, Y. Z.).

\section{Disclosure}

The authors report no conflicts of interest in this work.

\section{References}

1. Bray F, Ferlay J, Soerjomataram I, Siegel RL, Torre LA, Jemal A. Global cancer statistics 2018: GLOBOCAN estimates of incidence and mortality worldwide for 36cancers in 185 countries. CA Cancer J Clin. 2018;68(6):394-424. doi:10.3322/caac. 21492

2. Van Meir H, Kenter GG, Burggraaf J, et al. The need for improvement of the treatment of advanced and metastatic cervical cancer, the rationale for combined chemoimmunotherapy. Anticancer Agents Med Chem. 2014;14(2):190-203.

3. Sasano T, Mabuchi S, Kuroda H, et al. Predictors of survival in patients with FIGO stage IVB cervical cancer. Int J Gynecol Cancer. 2016;26(3):528-533. doi:10.1097/IGC.0000000000000642

4. Li H, Wu X, Cheng X. Advances in diagnosis and treatment of metastatic cervical cancer. J Gynecol Oncol. 2016;27(4):e43. doi:10.3802/jgo.2016.27.e43

5. Kim K, Cho SY, Kim BJ, Kim MH, Choi SC, Ryu SY. The type of metastasis is a prognostic factor in disseminated cervical cancer. $J$ Gynecol Oncol. 2010;21(3):186-190. doi:10.3802/jgo.2010.21.3.186

6. Wang Y, Farmer M, Izaguirre EW, et al. Association of definitive pelvic radiation therapy with survival among patients with newly diagnosed metastatic cervical cancer. JAMA Oncol. 2018;4(9):12881291. doi:10.1001/jamaoncol.2018.2677
7. Quinn MA, Benedet JL, Odicino F, et al. Carcinoma of the cervix uteri. FIGO 26th annual report on the results of treatment in gynecological cancer. Int J Gynaecol Obstet. 2006;95(Suppl 1):S43-S103. doi:10.1016/S0020-7292(06)60030-1

8. Carlson V, Delclos L, Fletcher GH. Distant metastases in squamouscell carcinoma of the uterine cervix. Radiology. 1967;88(5):961-966.

9. Hwang JH, Lim MC, Seo SS, Kang S, Park SY, Kim JY. Outcomes and toxicities for the treatment of stage IVB cervical cancer. Arch Gynecol Obstet. 2012;285(6):1685-1693.

10. Zighelboim I, Taylor NP, Powell MA, et al. Outcomes in 24 selected patients with stage IVB cervical cancer and excellent performance status treated with radiotherapy and chemotherapy. Radiat Med. 2006;24(9):625-630.

11. Liu FY, Yen TC, Chen MY, et al. Detection of hematogenous bone metastasis in cervical cancer: $18 \mathrm{~F}$-fluorodeoxyglucose-positron emission tomography versus computed tomography and magnetic resonance imaging. Cancer. 2009;115(23):5470-5480.

12. Deng K, Yang C, Tan Q, et al. Sites of distant metastases and overall survival in ovarian cancer: A study of 1481 patients. Gynecol Oncol. 2018;150(3):460-465.

13. Gibson AJW, Li H, D'Silva A, et al. Impact of number versus location of metastases on survival in stage IV M1b non-small cell lung cancer. Med Oncol. 2018;35(9):117.

14. Ning MS, Ahobila V, Jhingran A, et al. Outcomes and patterns of relapse after definitive radiation therapy for oligometastatic cervical cancer. Gynecol Oncol. 2018;148(1):132-138.

15. Hendriks LE, Derks JL, Postmus PE, et al. Single organ metastatic disease and local disease status, prognostic factors for overall survivalin stage IV non-small cell lung cancer: results from a populationbased study. Eur J Cancer. 2015;51(17):2534-2544.

16. Tangjitgamol S, Levenback CF, Beller U, Kavanagh JJ. Role of surgical resection for lung, liver, and central nervous system metastases in patients with gynecological cancer: a literature review. Int $J$ Gynecol Cancer. 2004;14(3):399-422.

17. Kim GE, Lee SW, Suh CO, et al. Hepatic metastases from carcinoma of the uterine cervix. Gynecol Oncol. 1998;70(1):56-60.

18. Mallekavu SB, Thanky AH, Kanakasetty GB, Kuntegowdanahalli L, Dasappa L, Jacob LA. Prognostic significance of bone only metastasis compared to visceral metastasis in patients with carcinoma cervix treated with platinum-based chemotherapy. South Asian $J$ Cancer. 2017;6(4):151-153.
Cancer Management and Research

\section{Publish your work in this journal}

Cancer Management and Research is an international, peer-reviewed open access journal focusing on cancer research and the optimal use of preventative and integrated treatment interventions to achieve improved outcomes, enhanced survival and quality of life for the cancer patient.
The manuscript management system is completely online and includes a very quick and fair peer-review system, which is all easy to use. Visit http://www.dovepress.com/testimonials.php to read real quotes from published authors. 